\title{
Los programas de incentivos podrían ser efectivos para dejar de fumar
}

\author{
Incentive programs for smoking cessation may be effective
}

\author{
Comentado de: \\ Notley C, et al. Cochrane Database Syst Rev. 2019 Jul \\ 17;7:CD004307. ${ }^{1}$
}

\section{Introducción}

Los incentivos financieros, monetarios o con vouchers (vales o cupones), se utilizan ampliamente para intentar inducir, reforzar y mantener cambios de comportamiento, incluido el abandono del tabaquismo. Se han utilizado en lugares de trabajo, en clínicas, hospitales y en programas comunitarios.

\section{Objetivos}

Determinar el efecto a largo plazo de los incentivos y los programas de manejo de contingencias (aquellos basados en técnicas para el control de estímulos y el refuerzo positivo) para dejar de fumar.

\section{Métodos de búsqueda}

Para esta actualización, los autores buscaron en el Registro Especializado del Grupo Cochrane de Adicción al Tabaco, en clinicaltrials.gov y en la Plataforma Internacional de Registro de Ensayos Clínicos (ICTRP). Las búsquedas más recientes se realizaron en julio de 2018.

\section{Criterios de selección}

Solo se consideraron ensayos aleatorizados que asignaron individuos, lugares de trabajo, grupos dentro de los lugares de trabajo o comunidades a algún esquema de incentivos para dejar de fumar comparado con un gupo control. Incluyeron estudios en ámbitos de población mixta (por ejemplo, basados en la comunidad, el trabajo, la clínica o la institución) y también estudios en embarazadas fumadoras.

\section{Recolección y análisis de datos}

Utilizaron métodos estándar del manual Cochrane. La medida de resultado primaria en los estudios de población mixta fue la abstinencia de fumar al seguimiento más prolongado (al menos seis meses desde el inicio de la intervención) o al menos al final del embarazo, en los ensayos de mujeres embarazadas. Cuando estuvieron disponibles, agruparon los datos de los resultados utilizando un modelo de efectos aleatorios, informando tasas de riesgo (Riesgo Relativo o RR) con sus intervalos de confianza (IC) del $95 \%$, utilizando estimaciones ajustadas para los ensayos aleatorizados por conglomerados (clusters). Se analizaron por separado los estudios realizados en poblaciones mixtas y los realizados en poblaciones de embarazadas.

\section{Resultados principales}

Treinta y tres estudios de población mixta cumplieron con los criterios de inclusión, incluyendo más de 21.600 participantes.
Los estudios se realizaron en ámbitos comunitarios, clínicas o centros de salud, lugares de trabajo y clínicas ambulatorias. Ocho estudios tuvieron bajo riesgo de sesgo, 10 tuvieron alto riesgo de sesgo y el resto fueron calificados como de riesgo de sesgo incierto. Veinticuatro de los ensayos se llevaron a cabo en los EE. UU., dos en Tailandia, uno en Filipinas, mientras que el resto habían sido realizados en países europeos. Los incentivos ofrecidos incluyeron pagos en efectivo o vales para productos $y$ comestibles, ofrecidos directamente o canjeables en línea. EI RR combinado para dejar de fumar con incentivos en el seguimiento más prolongado (seis meses o más) en comparación con los controles fue de 1,49 (IC95 \% 1,28 a 1,73; 31 ensayos, con un total de 20.097 participantes; heterogeneidad evaluada por $\mathrm{I}^{2}=33 \%$ ). Los resultados no fueron sensibles a la exclusión de seis estudios en los que se ofreció un incentivo para el cese tabáquico en el seguimiento a largo plazo (RR 1,40; IC95\% 1,16 a 1,69; 25 ensayos; 17,058 participantes; $I^{2}=36 \%$ ), sugiriendo que el impacto de los incentivos continúa por lo menos algún tiempo después de que cesen los incentivos.

Aunque no siempre se informaba claramente, el monto financiero total de los incentivos varió considerablemente entre los ensayos, desde cero (depósitos propios) hasta un rango entre 45 y 1.185 dólares. No hubo diferencias de efecto claras entre los ensayos que ofrecían incentivos de bajo o alto valor, ni los que fomentan los auto-depósitos reembolsables.

Se incluyeron 10 estudios con 2.571 mujeres embarazadas. Se consideró que dos estudios tenían bajo riesgo de sesgo, uno, alto riesgo y siete, riesgo incierto. Cuando se combinaron, los nueve ensayos con datos utilizables (ocho realizados en los EE. UU. y uno en el Reino Unido), obtuvieron un RR (hasta 24 semanas después del parto) de 2,38 (IC 95\%: 1,54 a 3,69; 2273 participantes; $\left.\left.\right|^{2}=41 \%\right)$, a favor de los incentivos.

\section{Conclusiones de los autores}

En general, existe evidencia de alta certeza de que los incentivos mejoran las tasas de abandono del tabaco durante el seguimiento a largo plazo, en estudios de población mixta. La efectividad de los incentivos parece mantenerse incluso cuando el último seguimiento ocurre después del retiro de los incentivos. También existe evidencia de certeza moderada, limitada por riesgos de sesgo, de que los esquemas de incentivos realizados entre las fumadoras embarazadas mejoran las tasas de abandono tabaco, tanto al final del embarazo como después del parto. Las investigaciones actuales y futuras podrían explorar diferencias más precisas entre los ensayos que ofrecen incentivos financieros de alto o bajo valor y auto-depósitos, dentro de una variedad de poblaciones de fumadores.

\section{Comentario}

En los últimos años se han publicado varias revisiones sistemáticas sobre incentivos para dejar de fumar. Giles et al incluyeron 16 estudios de cambio de comportamiento de salud incentivado, diez de los cuales se centraron en dejar de fumar y mostraron resultados consistentes ${ }^{2}$. Leeks et al mostraron un beneficio del $4,4 \%$ a una mediana de 12 meses de seguimiento con programas de cesación en el lugar de trabajo que incluían incentivos o competencias ${ }^{3}$. 
Otras dos revisiones sintetizan evidencia sobre esquemas de incentivos específicamente en mujeres embarazadas. Higgins et al resumieron una serie de seis ensayos de incentivos en fumadores embarazadas, con hallazgos son similares, calificando las intervenciones como prometedoras ${ }^{4}$. Una revisión Cochrane (Chamberlain 2017), evaluó la efectividad de las intervenciones psicosociales para dejar de fumar en el embarazo e incluyó un subconjunto de estudios que utilizaron incentivos junto con intervenciones alternativas ${ }^{5}$. Aunque su resultado primario fue la abstinencia al final del embarazo y la revisión actual contempló un mayor tiempo seguimiento, sus resultados fueron consistentes.

Podría suponerse que los ensayos de devolución de depósitos pueden propender a bajas tasas de aceptación en comparación con los programas basados en recompensas; sin embargo, las personas que se registran y aportan su propio dinero alcanzaron tasas de abandono comparables o más altas que los participantes que solo recibieron una recompensa.

Aunque se ha expresado cierta preocupación acerca de que las intervenciones basadas en incentivos atraigan a fumadores motivados más por las recompensas materiales que por el deseo de dejar de fumar, la tasa de abandono no fue insignificante y los niveles de engaño no variaron entre los grupos experimentales y de control.

Por otro lado, se necesitan más ensayos grandes y bien conducidos sobre la efectividad del uso de incentivos para dejar de fumar en países de ingresos bajos y medios y en mujeres embarazadas, incluyendo incentivos basados en depósitos. También sería necesario que se comparen de manera directa los incentivos altos vs. bajos, quizás operacionalizados como un porcentaje del ingreso medio de los participantes del estudio. Asimismo, la asequibilidad y la rentabilidad de los programas de incentivos debería probarse en el mundo real, como parte del proceso de evaluación, antes de recomendar su implementación.

\section{Conclusiones del comentador}

Existe moderada a alta calidad de evidencia de la efectividad de los incentivos incluso después de su retiro para dejar de fumar. La implementación y la aceptabilidad de este tipo de intervenciones en entornos del mundo real deben evaluarse formalmente, lo que incluye comparar o evaluar directamente el valor de los incentivos junto con otras intervenciones para dejar de fumar.

Agustín Ciapponi [ Centro Cochrane Argentino, Instituto de Efectividad Clínica y Sanitaria (IECS-CONICET) aciapponi@iecs.org.ar ]

Ciapponi A. Los programas de incentivos podrían ser efectivos para dejar de fumar. Evid Actual Pract Ambul. 2019;22(2):e002005. Comentado de: Notley C, Gentry S, Livingstone-Banks J, Bauld L, Perera R, Hartmann-Boyce J. Incentives for smoking cessation. Cochrane Database Syst Rev. 2019 Jul 17;7:CD004307. PMID CD004307

\section{Referencias}

1. Notley C, Gentry S, Livingstone-Banks J, Bauld L, Perera R, Hartmann-Boyce J. Incentives for smoking cessation. Cochrane Database of Systematic Reviews. 2019;Available from: 10.1002/14651858.CD004307.pub6.

2. Giles EL, Robalino S, McColl E, Sniehotta FF, Adams J. The effectiveness of financial incentives for health behaviour change: systematic review and meta-analysis. PLoS One. 2014;9:e90347. Available from: 10.1371/journal.pone.0090347.

3. Leeks KD, Hopkins DP, Soler RE, Aten A, Chattopadhyay SK, on Community Preventive STF. Worksite-based incentives and competitions to reduce tobacco use. A systematic review. Am J Prev Med. 2010;38:S263-74. Available from: 10.1016/j.amepre.2009.10.034.

4. Higgins ST, Washio Y, Heil SH, Solomon LJ, Gaalema DE, Higgins TM, et al. Financial incentives for smoking cessation among pregnant and newly postpartum women. Prev Med. 2012;55 Suppl:S33-40. Available from: 10.1016/j.ypmed.2011.12.016.

5. Chamberlain C, OḾara-Eves A, Porter J, Coleman T, Perlen SM, Thomas J, et al. Psychosocial interventions for supporting women to stop smoking in pregnancy. Cochrane Database Syst Rev. 2017;2:CD001055. Available from: 10.1002/14651858.CD001055.pub5. 